\title{
When is neuroimaging appropriate in children with headaches?
}

\author{
Luis S. Medina
}

(C) Springer-Verlag 2011

\section{Summary of evidence}

Determination of the appropriateness of imaging is made based on the frequency, pattern, family history and associated seizure or neurological findings (Table 1).

These guidelines reinforce the primary importance of careful acquisition of the medical history and performance of a thorough examination, including a detailed neurological examination. Among children at risk for brain lesions based on these signs and symptoms, neuroimaging with either MR imaging or CT is valuable in combination with close clinical follow-up.

Table 1 Suggested guidelines for neuroimaging in pediatric patients with headaches

Persistent headaches of less than 1 month duration

Headaches associated with abnormal neurological examination

Headaches associated with seizures

Headaches with new onset of severe episodes or change in the type of headache

Persistent headaches without family history of migraine

Family or medical history of disorders that may predispose one to CNS lesions, and clinical or laboratory findings that suggest CNS involvement

Disclaimer Dr. Medina has no financial interests, investigational or off-label uses to disclose.

L. S. Medina $(\bowtie)$

Miami Children's Hospital,

Miami, FL 33155, USA

e-mail: santiago.medina@mch.com

\section{What is the cost-effectiveness of neuroimaging in patients with headaches?}

A cost-effectiveness-analysis study assessed the clinical and economic consequences of three diagnostic strategies in the evaluation of children with headache suspected of having a brain tumor: (1) MR imaging, (2) CT followed by MR imaging for positive results (CT-MR imaging) and (3) no neuroimaging with close clinical follow-up. This model suggests that MR imaging maximizes quality-adjusted life years (QALY) gained at a reasonable cost-effectiveness ratio in patients at high risk of having a brain tumor. Conversely, the strategy of no imaging with close clinical follow-up is cost-saving in low-risk children. Although the CT-MR imaging strategy maximizes QALY gained in the intermediate-risk patients, its additional cost per QALY gained is high. In children with headaches, appropriate selection of patients and diagnostic imaging strategies can maximize quality-adjusted life expectancy and decrease costs of medical work-up. 